\section{AVALIAÇÃO DO RISCO DE DESENVOLVER DIABETES MELLITUS TIPO 2 EM POPULAÇÃO UNIVERSITÁRIA}

\author{
Assessment of risk of developing type 2 diabetes mellitus in a \\ university population
}

\author{
Evaluación del riesgo de desarrollo de Diabetes Mellitus del \\ tipo 2 en la población universitaria
}

\section{RESUMO}

Objetivo: Verificar a prevalência dos fatores de risco para desenvolver diabetes mellitus tipo 2 (DM2) em uma população universitária. Métodos: Estudo observacional, transversal, realizado entre 2013 e 2014 em uma universidade de Parnaíba-PI, com indivíduos maiores de 18 anos que estudam ou trabalham no campus. Do total populacional disposto no campus (4.310 pessoas), participaram da pesquisa 74\% (111) dos docentes (G1), 77,68\% (94) dos técnicos administrativos (G2) e 32,10\% (1.299) dos discentes, totalizando 1.504 indivíduos que responderam ao questionário "Are you at risk for type 2 diabetes?", traduzido para a língua portuguesa, de forma impressa, individual e anônima, com as respostas obtidas através de autopreenchimento. Calculou-se o risco de desenvolver DM2 para cada grupo, a associação entre o grupo e o risco de DM2 com teste do Qui-Quadrado $(p<0,05)$ e o risco relativo (RR) para desenvolvimento de DM2 considerando os escores obtidos no grupo. Resultados: A amostra contemplou 34,89\% (1504) do n amostral. O risco de DM2 foi apresentado por 16,21\% (18) dos indivíduos do G1, 13,82\% (13) do G2 e 1,23\% (16) do G3. Quanto à hipertensão, verificou-se a incidência de $17,1 \%$ (19) no G1, 12,8\% (12) no G2 e 5,9\% (77) no G3. Nos G1, G2 e G3, 59,5\% (66), 38,3\% (36) e 41,26\% (536), respectivamente, não eram fisicamente ativos. O risco de desenvolver DM2 foi elevado no G1 e no G2, significativamente diferente do G3. Conclusão: Encontrou-se a prevalência do risco de desenvolver diabetes mellitus tipo 2 (DM2) de 16,21\% no grupo de docentes, $13,82 \%$ no grupo de técnicos administrativos e $1,23 \%$ no grupo de discentes, destacando-se a obesidade e a inatividade física como fatores de risco mais comuns.

Descritores: Fatores de Risco; Diabetes Mellitus Tipo 2; Universidades; Questionários.

\section{ABSTRACT}

Objective: To determine the prevalence of risk factors for developing type 2 diabetes mellitus (T2DM) in a university population. Methods: Observational, cross-sectional study, carried out between 2013 and 2014, in a university of Parnaiba, Piaui State, with individuals aged over 18 years, who study or work in campus. Of the total campus population provision (4,310 individuals), participated in the survey $74 \%$ (111) of teachers (G1), 77.68\% (94) of the administrative staff $(G 2)$, and $32.10 \%(1,299)$ of the students, totalling 1,504 individuals who answered the questionnaire "Are you at risk for type 2 diabetes?", translated into Portuguese, in a written, individual, and anonymous form, with responses obtained through self- administration. The risk of developing T2DM was calculated for each group, as well as the association between the group and the T2DM risk, using the chi-square test ( $p<0.05)$, and the relative risk of T2DM development, considering the scores obtained in the group. Results: The sample included 34.89\% (1504) of the total sample $n$. T2DM risk was presented by $16.21 \%$ (18) of the individuals in G1, 13.82\% (13) of G2, and 1.23\% (16) of G3. For hypertension, the incidence of $17.1 \%$ (19) in $G 1,12.8 \%$ (12) in $G 2$, and $5.9 \%$ (77) in G3 was found. In G1, G2 and G3, 59.5\% (66), 38.3\% (36), and 41.26\% (536), respectively, were not physically active. The risk of developing T2DM was higher in G1 and G2, and
Artigo Original

\author{
Alessandra Tanuri Magalhães ${ }^{(1)}$ \\ Baldomero Antonio Kato da \\ Silva ${ }^{(1)}$ \\ Jéssica Alves Ribeiro( ${ }^{(1)}$ \\ Joaquim Fontes de Aguiar \\ Bisneto $^{(1)}$ \\ Lana Priscilla Ibiapina Pereira ${ }^{(1)}$ \\ Nádia Veras Machado(1) \\ Cristiano Sales da Silva ${ }^{(1)}$ \\ Vinicius Saura Cardoso ${ }^{(1)}$
}

1) Universidade Federal do Piauí - UFPI Parnaíba (PI) - Brasil 
significantly different from $G 3$. Conclusion: The prevalence of risk of developing type 2 diabetes was found at $16.21 \%$ in the teachers group, $13.82 \%$ in the administrative staff group, and $1.23 \%$ in the students group, with obesity and physical inactivity evidenced as the most common risk factors.

Descriptors: Risk Factors; Diabetes Mellitus, Type 2; Universities; Questionnaires.

\section{RESUMEN}

Objetivo: Verificar la prevalencia de los factores de riesgo para el desarrollo de la Diabetes Mellitus tipo 2 (DM2) en una población universitaria. Métodos: Estudio observacional y transversal realizado entre 2013 y 2014 en una universidad de Parnaiba-PI con individuos mayores de 18 años que estudian o trabajan en el campus. Del total poblacional del campus (4.310 personas), participaron de la investigación el 74\% (111) de los docentes (G1), el 77,68\% (94) de los técnicos administrativos (G2) y el 32,10\% (1.299) de los discentes en un total de 1.504 individuos que contestaron el cuestionario "Are you at risk for type 2 diabetes?", que fue traducido para el idioma portugués, de forma impresa, individual y anónima con las respuestas obtenidas a través de auto aplicación. Se calculó el riesgo de desarrollo de la DM2 en cada grupo, la asociación del grupo y el riesgo de DM2 con la prueba de Chi-cuadrado $(p<0,05)$ y el riesgo relativo $(R R)$ para el desarrollo de la DM2 considerando las puntuaciones obtenidas en el grupo. Resultados: La muestra incluyó el 34,89\% (1504) del cálculo total de la muestra. El riesgo de la DM2 se diós en el 16,21\% (18) de los individuos del G1, el 13,82\% (13) del G2 y el $1,23 \%$ (16) del G3. Sobre la hipertensión, se verifico la incidencia del 17,1\% (19) en el G1, el 12,8\% (12) en el G2 y el 5,9\% (77) en el G3. En el G1, G2 y G3, el 59,5\% (66), el 38,3\% (36) y el $41,26 \%$ (536), respectivamente, no eran físicamente activos. El riesgo del desarrollo de la DM2 fue elevado en el G1 y el G2, significativamente distinto del G3. Conclusión: Se encontró una prevalencia del riesgo de desarrollo de la Diabetes Mellitus tipo 2 (DM2) del 16,21\% en el grupo de docentes, del 13,82\% en el grupo de técnicos administrativos y del 1,23\% en el grupo de discentes destacándose la obesidad y la inactividad fisica como factores de riesgo más comunes.

Descriptores: Factores de Riesgo; Diabetes Mellitus Tipo 2; Universidades; Cuestioarios.

\section{INTRODUÇÃO}

Diabetes mellitus (DM) é uma doença metabólica de etiologia múltipla, caracterizada por hiperglicemia, com alteração na secreção, na ação de insulina ou ambas ${ }^{(1)}$. Pode ser classificada em duas categorias: DM tipo 1 (DM1), representando apenas $5-10 \%$ das pessoas com diabetes, estando relacionada à deficiência de insulina por destruição autoimune das células-B do pâncreas; e DM tipo 2 (DM2), representando $90-95 \%$ das pessoas com diabetes, resultado de um complexo processo fisiopatológico que culmina com a resistência insulínica ${ }^{(1,2)}$.

A DM2 é um importante problema de saúde pública global. A Federação Internacional de Diabetes indica que o número de pessoas com diabetes irá aumentar de 285 milhões em 2010 para 438 milhões em 2030, com mais de $70 \%$ dos casos acontecendo em países em desenvolvimento. Entre eles, estão a China, Índia, Rússia, Brasil, Paquistão, Indonésia e Bangladesh. Nesses países, quase 80\% das mortes estão relacionadas à diabetes ${ }^{(3)}$. Em 2012, a prevalência estimada para o Brasil foi de $10,3 \%{ }^{(4)}$.

A DM2 é uma das principais doenças crônicas que afetam o homem contemporâneo. Dentre fatores responsáveis, destacam-se: história familiar de DM2, urbanização, estilo de vida, dieta inadequada, sedentarismo, consumo de álcool e hipertensão arterial. A esses fatores, acrescentam-se a idade, o sexo e a taxa de glicemia capilar elevada $^{(5)}$.

Há uma década, a obesidade é considerada o principal fator para o aparecimento de doenças metabólicas como a $\mathrm{DM}^{(6)}$. Os índices de obesidade vêm crescendo de forma alarmante, fazendo com que autoridades de saúde estimulem programas que favoreçam a redução do índice de massa corpórea (IMC) na população ${ }^{(7)}$. Uma das maneiras sugeridas para isso são programas de atividade física, pois, além de favorecerem a diminuição do risco de DM, também melhoram a capacidade cardiovascular de quem pratica, diminuindo o risco de doenças cardíacas, tão comuns na população com $\mathrm{DM}^{(8)}$.

Diretrizes atuais têm defendido a aplicação de escores de riscos multivariados a fim de prever o risco para DM2 em indivíduos saudáveis ${ }^{(9)}$. Esses escores de risco servem para classificar indivíduos com precisão e direcionar as intervenções de prevenção. A coleta de dados pode ser feita a partir de questionários, os quais provalvemente são menos onerosos e mais aceitáveis do que os métodos bioquímicos de triagem, como a medição de glicose no sangue ${ }^{(10)}$.

A elaboração de programas de prevenção de diabetes para determinado público deve ser delineada a partir das características da população estudada, para que, dessa forma, os objetivos sejam atingidos, modificando os aspectos apresentados como fatores de risco ${ }^{(11)}$. A utilização de questionários também se mostra eficiente por atingir um grande número de pessoas devido à praticidade de aplicação e resposta ${ }^{(12)}$.

Existe uma tendência de se encontrar adultos jovens vulneráveis a desenvolver DM2 em campi universitários, pois, com a busca por estabilidade profissional, eles aderem ao sedentarismo e ao sobrepeso, influenciados pela evolução 
tecnológica que minimiza o esforço físico nas atividades cotidianas e pela alimentação rápida e prática ${ }^{(13)}$.

As empresas, de modo geral, estão discutindo e adaptando-se para receber cada vez mais colaboradores com DM2, pois, apesar de todas as pesquisas referente à prevenção e tratamento, existe uma epidemia de diabetes ocorrendo no mundo ${ }^{(14)}$.

Apesar de o número de diagnóstico de DM2 aumentar anualmente, muitas pessoas podem possuir a doença, porém, devido ao não aparecimento de sintomas ou estes serem de baixa intensidade, negligenciam a necessidade de exames e avaliações frequentes. Além disso, por ter características vastamente estudadas e, dessa forma, conhecidas, métodos de identificação de população de risco para desenvolver DM2 são de extrema importância para tentar elaborar políticas de prevenções direcionadas ${ }^{(11)}$.

Partindo dessa premissa, o estudo objetivou verificar a prevalência dos fatores de riscos para desenvolver diabetes mellitus tipo 2 (DM2) em uma população universitária.

\section{MÉTODOS}

Realizou-se um estudo observacional do tipo corte transversal entre os meses de setembro de 2013 e fevereiro de 2014, na Universidade Federal do Piauí (UFPI), campus Ministro Reis Velloso, em Parnaíba-PI.

Incluíram-se na amostra indivíduos maiores de 18 anos que estudavam ou trabalhavam no campus de Parnaíba e que haviam aceitado participar do estudo. Os indivíduos com diagnóstico clínico de diabetes mellitus tipo 2 foram excluídos. Os participantes receberam esclarecimentos quanto aos objetivos e procedimentos da pesquisa, e apenas receberam os questionários após a assinatura do Termo de Consentimento Livre e Esclarecido (TCLE).

Atualmente, o campus universitário de Parnaíba constitui-se de 11 cursos: Administração de Empresas, Biomedicina, Ciências Contábeis, Ciências Econômicas, Engenharia de Pesca, Fisioterapia, Licenciatura em Biologia e Matemática, Pedagogia, Psicologia e Turismo, contendo um total de 4.039 discentes, 150 docentes e 121 técnicos.

Dividiu-se a população em três grupos, conforme as atividades realizadas no campus: grupo 1 - docentes (G1), grupo 2 - técnicos administrativos (G2) e grupo 3 discentes (G3).

Calculou-se a amostra necessária para o estudo através de amostragem aleatória simples, sem reposição, considerando-se um nível de confiança de $95 \%$ e erro de estimação de $5 \%$, resultando em um mínimo de 353 participantes para a realização do estudo.
Do total populacional disposto no campus, participaram da pesquisa $111(74 \%)$ do G1, $94(77,68 \%)$ do G2 e 1.299 $(32,10 \%)$ do G3, totalizando 1.504 indivíduos. A distribuição percentual da amostra aproximou-se da distribuição da população nos grupos de divisão dentro do campus, sendo observado ao final $38 \%(\mathrm{n}=111)$ da amostra pertencente ao $\mathrm{G} 1,6,25 \%$ (n=94) ao G2 e 86,36\% (1299) ao G3.

Utilizou-se o questionário "Are you at risk for type 2 diabetes?" ("Você tem risco de ter diabetes tipo 2?"), da Sociedade Americana de Diabetes, adaptado ${ }^{(15)}$, que passou por processo de tradução e adaptação cultural, seguindo sugestões $^{(16,17)}$ para a validação e utilização do questionário proposto. A versão original do questionário (Figura 1) foi traduzida para o português de forma independente, gerando duas versões. Os pesquisadores instruíram os tradutores (ambos fisioterapeutas e docentes) a elaborarem um relatório sobre dúvidas e dificuldades. Um terceiro tradutor, não pertencente à área acadêmica, sintetizou as duas traduções e comparou com a versão original do questionário, gerando outra versão em língua portuguesa.

\section{ARE YOU AT RISK FOR

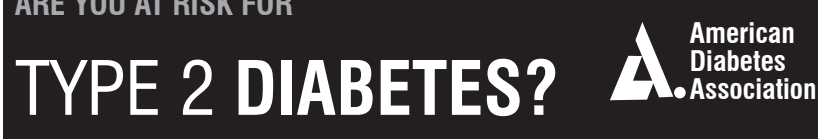

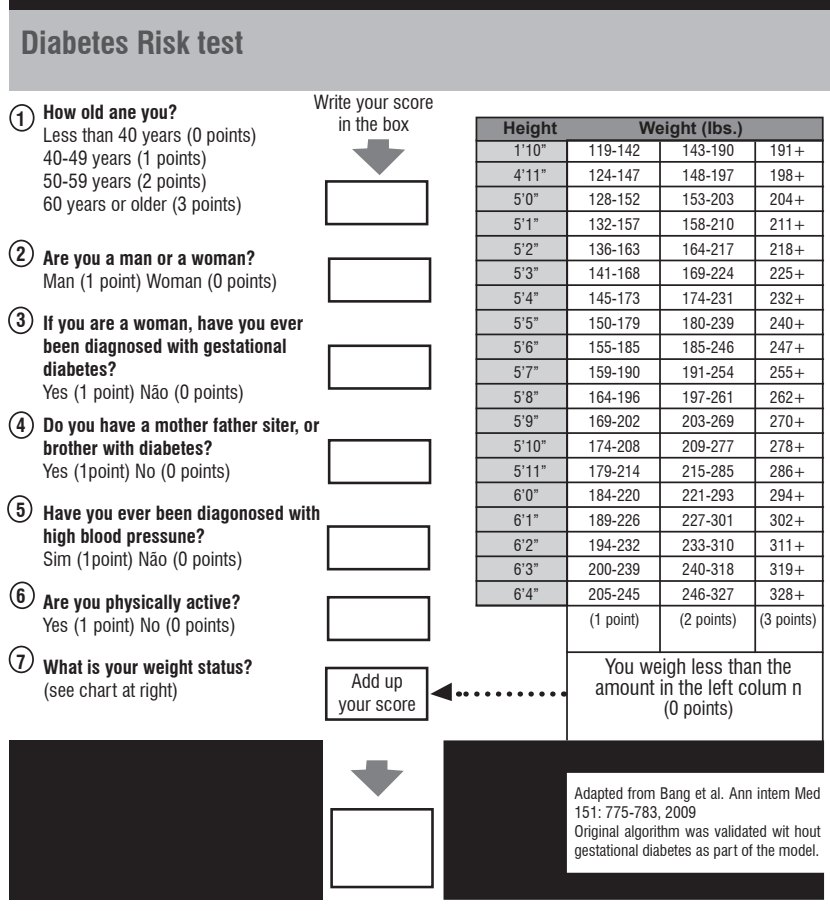

Figura 1 - Questionário original “Are you at risk for type 2 diabetes?", da Sociedade Americana de Diabetes. (http:// www.diabetes.org/) 


\section{VOCÊ TEM RISCO DE TER

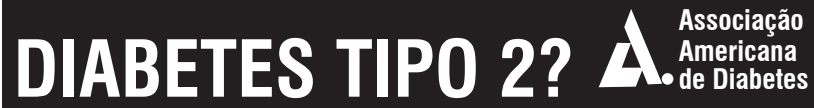

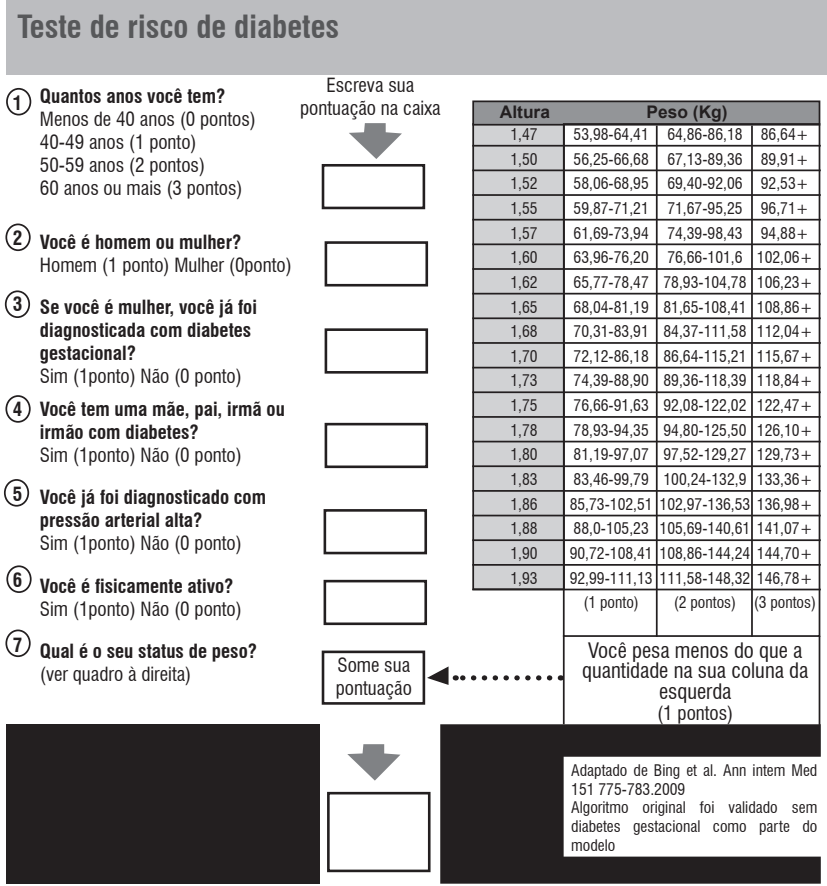

Figura 2 - Versão final brasileira do "Are you at risk for type 2 diabetes?”. Teresina-PI, 2013-2014.

Retrotraduziu-se esta versão por dois outros tradutores independentes, sem qualquer conhecimento da versão original. O primeiro tradutor não tinha conhecimento sobre a área médica e não foi informado sobre os objetivos do estudo. O segundo tradutor era fisioterapeuta, docente em uma universidade norte-americana e conhecedor dos conceitos que seriam avaliados, porém não lhe foram transmitidas informações sobre o objetivo do estudo.

Posteriormente, a tradução foi revisada pelos pesquisadores, pelos tradutores e por dois fisioterapeutas não ligados à presente pesquisa para verificar a clareza, relevância, coerência e significados dos itens em relação ao original. Por fim, todos os revisores aprovaram a versão final em língua portuguesa (Figura 2).

Com base nas instruções do questionário aplicado em sua versão final, consideraram-se como risco de desenvolvimento de DM2 as somatórias de pontos que atingissem escore maior ou igual a cinco (5).

Aplicaram-se os questionários de forma individual, anônima, com as respostas obtidas através de autopreenchimento. Eles eram disponibilizados em meio impresso aos participantes, os quais eram orientados a ler cuidadosamente as instruções para preenchimento e responder conforme o entendimento destas.

Consideraram-se válidos os questionários em perfeito estado (sem danos, desenhos ou rasuras), respondidos apenas na presença dos pesquisadores. Os questionários rasurados ou incompletos eram desconsiderados na tabulação dos dados.

A coleta dos dados ocorreu de acordo com o grupo avaliado. Realizou-se a visita aos discentes e docentes pelos pesquisadores. Após apresentação e esclarecimentos sobre a pesquisa e seus procedimentos, os indivíduos receberam os TCLE e logo após os questionários para preenchimento, dispondo de tempo livre para registro das respostas.

Os integrantes do G2 foram convidados à participação no estudo em seus locais de trabalho dentro do campus, de acordo com a lotação exercida no momento do convite, recebendo orientações e preenchendo os questionários de forma idêntica aos demais grupos pesquisados.

Após o preenchimento, os questionários eram imediatamente entregues aos pesquisadores, que os armazenavam em pastas identificadas apenas pelo grupo pesquisado. Ao final do período de coleta, calculou-se o risco de DM2 para cada indivíduo.

Calculou-se a representatividade dos indivíduos por grupo com risco de desenvolver DM2, sendo esta expressa em frequência de aparecimento e porcentagem. Para verificação da associação entre o grupo e o risco de DM2, utilizou-se o teste do Qui-Quadrado, considerando-se o nível de significância $p<0,05$. Posteriormente, analisouse cada questão de forma individualizada para identificar quais fatores se destacam no aumento do risco de DM2 na população estudada. Calculou-se o risco relativo (RR) para desenvolvimento de DM2, considerando-se os escores obtidos nos grupos amostrais. Para os cálculos amostrais e análises estatísticas, utilizou-se o programa BioEstat 5.0.

O estudo obteve aprovação do Comitê de Ética em Pesquisa envolvendo Seres Humanos da Universidade Federal do Piauí (parecer $\mathrm{n}^{0}$ 676.235) e todos os procedimentos foram realizados em concordância com a Resolução 466/12 do Conselho Nacional de Saúde.

\section{RESULTADOS}

A amostra estudada contemplou 1.504 participantes, perfazendo $34,89 \%$ do número amostral total (4.310) do campus Ministro Reis Velloso da UFPI. Especificamente, aplicou-se o questionário em $74 \%$ (111) do grupo de docentes, 77,68\% (94) do grupo de técnicos administrativos e $32,1 \%$ (1299) do grupo de discentes. 


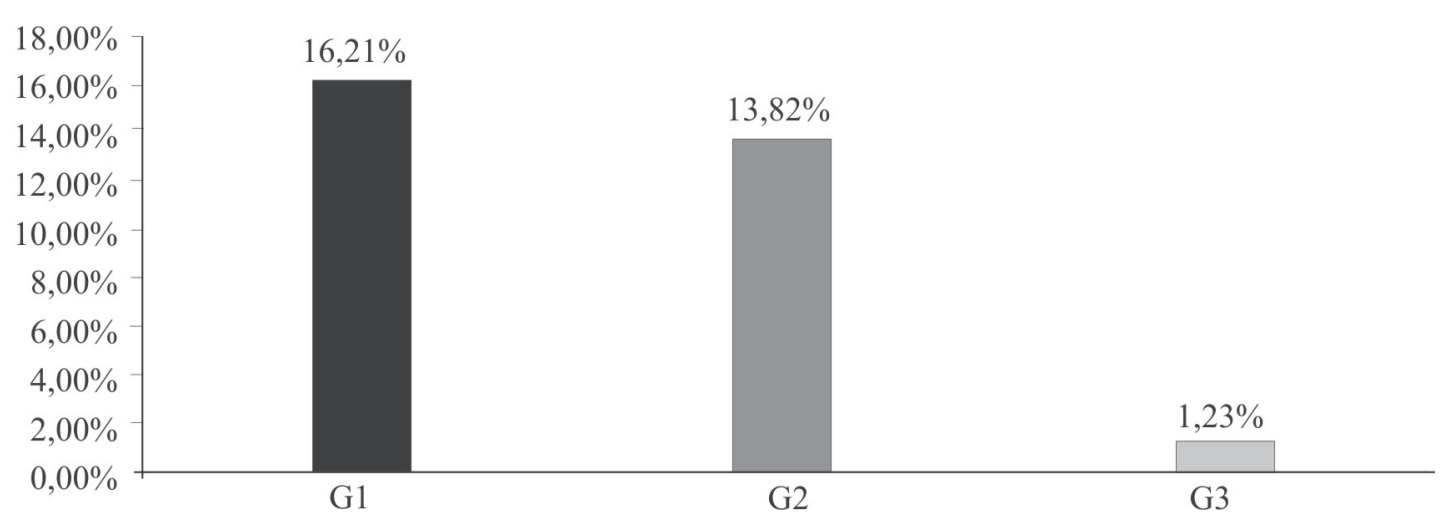

Figura 3 - Risco em desenvolver diabetes mellitus tipo 2 para os grupos G1 (docentes), G2 (técnicos administrativos) e G3 (discentes). Parnaíba-PI, 2013-2014.

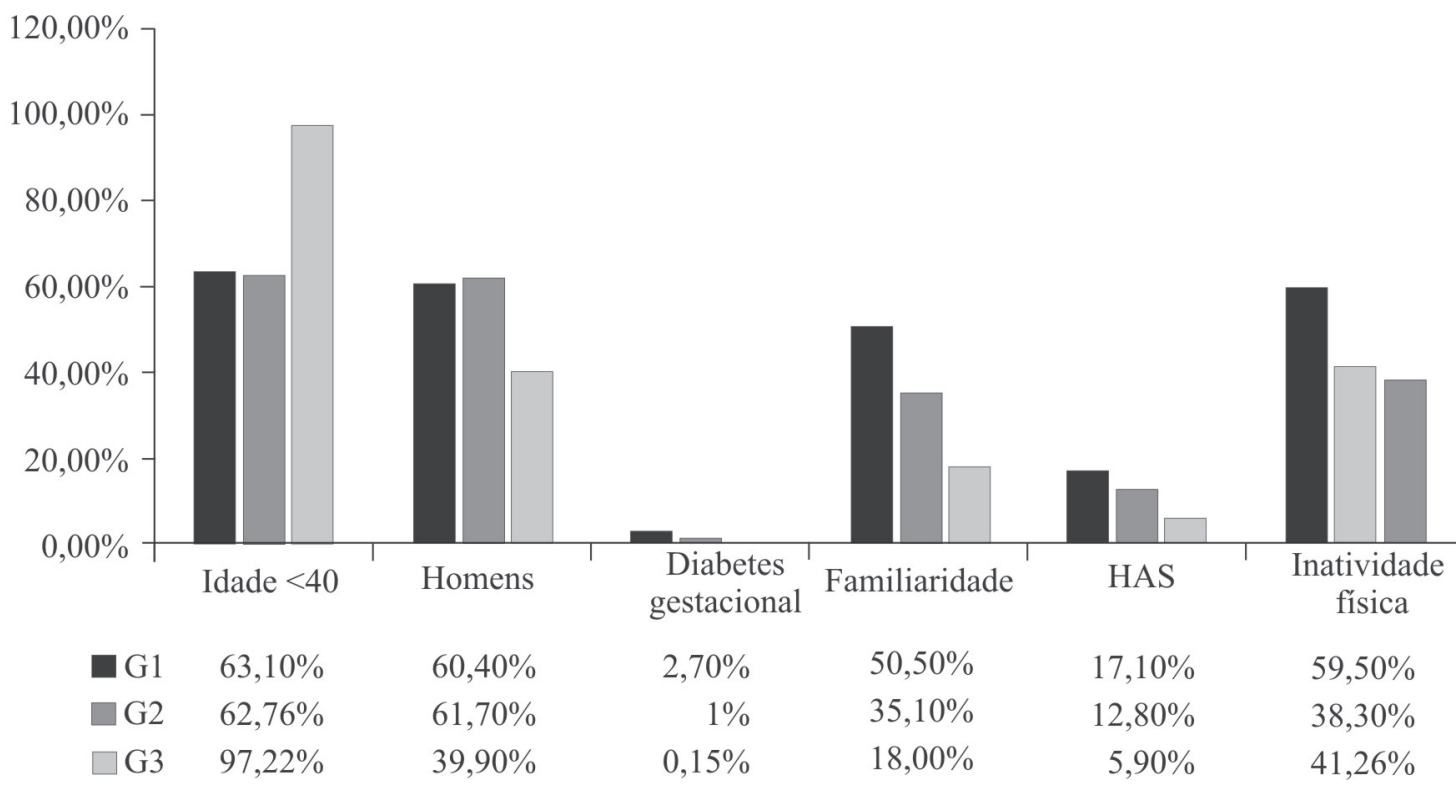

Figura 4 - Fatores de risco em desenvolver diabetes mellitus tipo 2 para os grupos G1 (docentes), G2 (técnicos administrativos) e G3 (discentes). Parnaíba-PI, 2013-2014.

Dezoito indivíduos do G1 (16,21\%), 13 do G2 (13,82\%) e 16 do G3 (1,23\%) apresentaram risco de DM2 (Figura 3). O teste do Qui-Quadrado mostrou haver associação entre os grupos analisados e o risco de DM2 $(\mathrm{p}<0,0001)$.

Quando comparados isoladamente, não se observou associação entre os grupos G1 e G2 (p=0,6830). A comparação entre os grupos G1 e G3 e entre os grupos G2 e G3 mostrou associação significativa para risco de DM2 $(\mathrm{p}<0,0001)$.

$\mathrm{Na}$ análise isolada de cada questão, a porcentagem dos fatores de risco foi estabelecida por grupo. Inicialmente, quanto à idade, todos os grupos eram compostos majoritariamente por indivíduos adultos jovens, com idade inferior a 40 anos (G1 - 63,1\% (69), G2 - 70,2\% (66) e G3 - 97,22\% (1263)).

Quanto ao gênero, o questionário utilizado oferece 1 ponto para o sexo masculino, sendo verificado que no G1 60,4\% (67), no G2 61,7\% (58) e no G3 39,9\% (518) eram homens.

A incidência de diabetes gestacional durante a gestação das participantes do sexo feminino mostrou-se pequena para todos os grupos estudados (G1 - 2,7\% (3), G2 - 1,1\% (1) e G3 - 0,15\% (2)). 
Quanto ao fator de risco relacionado à familiaridade, a ocorrência no G1 foi de 50,5\% (56), no G2 de 35,1\% (33) e no G3 de 18\% (234).

Referente ao diagnóstico de hipertensão arterial sistêmica (HAS), pôde-se verificar a incidência de 17,1\% (19) no G1, 12,8\% (12) no G2 e 5,9\% (77) no G3.

Quando pesquisada a realização de atividade física nos participantes, pontuando o indivíduo inativo fisicamente, encontraram-se os valores apresentados pelo G1 de 59,5\%
(66), no G2 de 38,3\% (36) e no G3 de 41,26\% (536) (Figura 4).

O status de peso recebe a pontuação de 0 a 3, seguindo ordem crescente de risco. Para o G1, 66,66\% (74) receberam pontuação, sendo 10,81\% (12) com 2 pontos. $\mathrm{O}$ G2 teve $62,76 \%$ (59) dos seus participantes pontuando no questionário, sendo $14,89 \%$ (14) com 2 pontos. Já no G3 $46,14 \%$ (599) dos participantes pontuaram, porém apenas $4,5 \%$ (58) com 2 pontos.

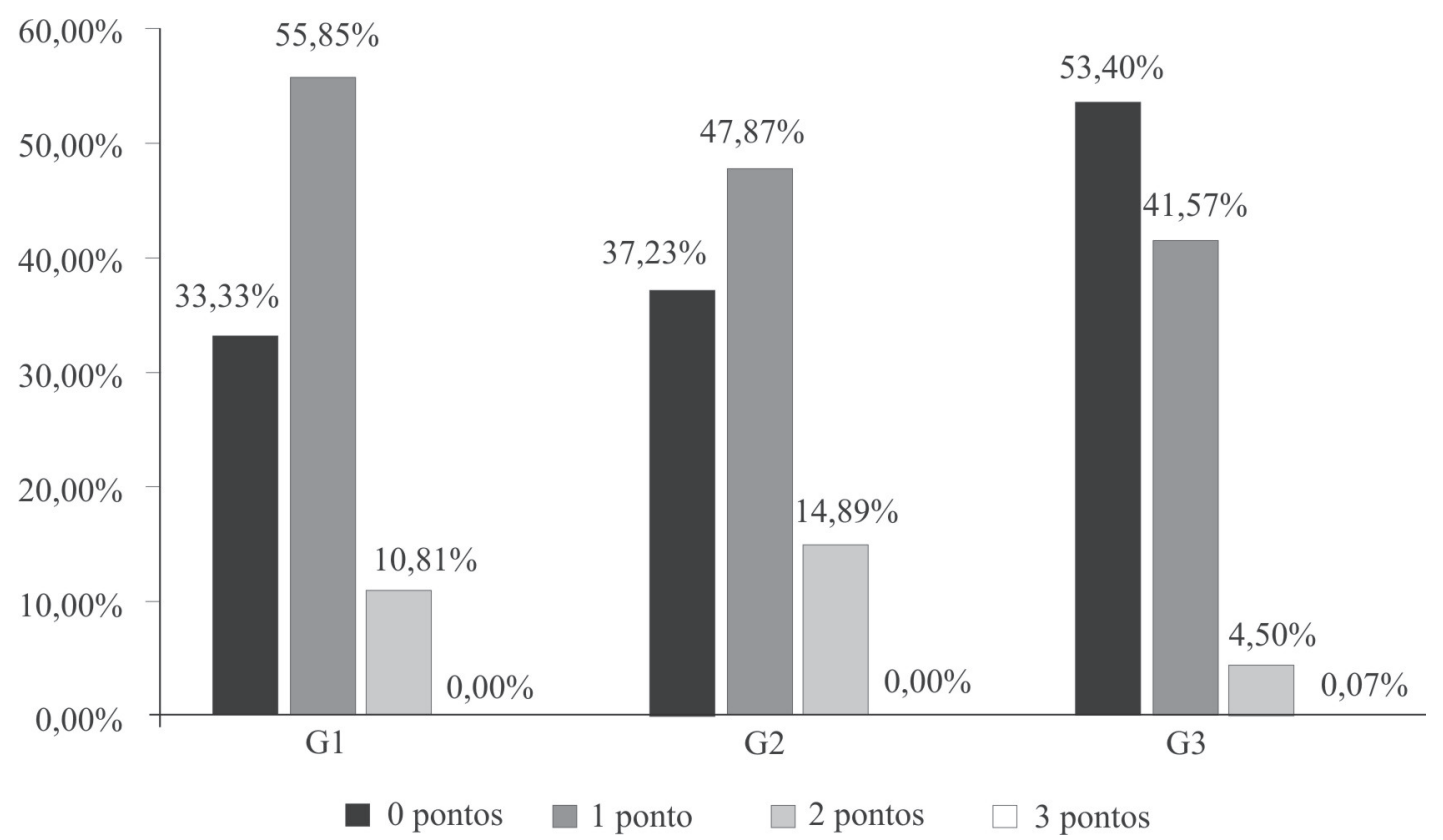

Figura 5 - Status de peso para os grupos G1 (docentes), G2 (técnicos administrativos) e G3 (discentes). Parnaíba-PI, 2013-2014.

Apenas o G3 possuiu participantes que somaram 3 pontos $(0,07 \%(1))$, aumentando o fator de risco para DM2 desse grupo (Figura 5).

\section{DISCUSSÃO}

O DM2 faz parte das chamadas doenças crônicas não transmissíveis (DCNT), que produzem uma carga econômica com elevados custos para os sistemas de saúde e previdenciários, em decorrência da mortalidade e invalidez precoces, afetando a sociedade, as famílias e as pessoas portadoras dessas doenças ${ }^{(18)}$. A população adulta jovem é um dos principais focos do Plano de Ações Estratégicas para o Enfrentamento das DCNTs no Brasil 2011-2022 $2^{(19)}$.
O referido plano tem como um dos seus eixos a promoção da saúde que valoriza ações populacionais, e estas podem contribuir para a redução da incidência das DCNTs e são custo-efetivas $^{(20)}$.

Assim, a averiguação de fatores potenciais que proporcionam informações quanto ao risco de desenvolvimento das DCNTs e, no presente estudo, do DM é fundamental para o planejamento das ações em educação e promoção voltadas para a saúde dessa população, sendo os integrantes de universidades, professores, técnicos e alunos uma amostra importante para ser estudada.

Nos últimos anos, o número de professores, alunos e técnicos administrativos no ensino superior vem aumentando no Brasil com a expansão recente das universidades federais 
brasileiras, a reestruturação da infraestrutura e a adesão da totalidade das 54 instituições federais de ensino superior. Isso implicou na criação de dez novas universidades federais em todas as regiões, e na criação e consolidação de 49 campi universitários. Com relação à oferta de vagas, entre o ano de 2006 e 2010, foram criadas 77.279 vagas e 1.035 novos cursos de graduação presencial ${ }^{(21)}$.

Observa-se que os frequentadores da universidade passam o dia nesse local e, dessa forma, realizam suas atividades de trabalho e estudo, refeições e, às vezes, atividades físicas e culturais. Dessa forma, o ambiente universitário torna-se propício para realizar estudos com o objetivo de identificar riscos de saúde na população que frequenta a universidade por diversos motivos, como transmitir informação e conteúdo, adquirir conhecimento ou dar suporte para que o ensino ocorra ${ }^{(21)}$.

No presente estudo, utilizou-se a versão final em língua portuguesa do questionário "Are you at risk for type 2 diabetes?", adaptado ${ }^{(15)}$. O questionário foi considerado de linguagem simples e clara pelos autores do presente estudo, visto que a tradução e retrotradução eram idênticas, mesmo quando realizadas de forma independente.

A população estudada na presente pesquisa mostrouse significativa, atingindo $34,89 \%$ do total da população do campus de Parnaíba da UFPI, satisfazendo o calculo amostral. Pode-se verificar o risco de desenvolver diabetes tipo 2 significativamente diferente no G1 $(16,21 \%)$ e G2 $(13,82 \%)$ em relação ao G3 $(1,23 \%)$.

Não há muitos relatos na literatura sobre o risco de DM2 dentro do ambiente universitário. Um estudo ${ }^{(22)}$ aplicou um questionário elaborado pelos próprios autores investigando variáveis socioeconômicas (idade, sexo, semestre, curso, renda familiar e estado civil), histórico familiar de DCNTs (hipertensão arterial, diabetes mellitus e obesidade), frequência de atividade física, uso de suplemento alimentar (quantidade e indicação) e hábitos alimentares (frequência de consumo de frutas, sucos, legumes, verduras, leites e derivados) em 383 estudantes da área da saúde, com idade média de 23,8 anos. Identificaram que $60,1 \%$ dos estudantes possuíam na família histórico de HAS, $46,2 \%$ histórico de DM2 e 33,4\% histórico de obesidade.

Em países emergentes, há uma tendência de aumento na frequência de $\mathrm{DM}$ em todas as faixas etárias, especialmente nas mais jovens (abaixo de 40 anos) ${ }^{(23)}$. Isso não ocorreu no presente estudo. Tal tendência ocorre devido ao aumento dos fatores de risco de sobrepeso e de doenças cardiovasculares, como índice de massa corporal (IMC) variando de 26-38 $\mathrm{kg} / \mathrm{m} 2$, pressão arterial e triacilgliceróis elevados ${ }^{(24)}$.

Analisando individualmente os fatores abordados no questionário, pode-se verificar no presente estudo que o risco de DM2 no G3 é pequeno, pois teve a maioria de seus componentes com idade inferior a 40 anos $(97,22 \%)$, visto que são estudantes e geralmente entram nas universidades por volta dos 18 aos 22 anos, com baixa incidência de diabetes gestacional (18\%) e hipertensão arterial sistêmica - HAS (5,9\%); e 53,40\% da população do grupo estava com peso adequado, segundo o questionário utilizado. Dessa forma, o risco em desenvolver DM2 é baixo, pois a glicemia basal aumenta progressivamente com a idade, assim como a incidência de $\mathrm{HAS}^{(24)}$.

Analisando as respostas dos questionários no G1 e G2, pode-se verificar que a maioria dos voluntários está abaixo de quarenta anos $(63,1 \%$ e $70,2 \%)$, pertence ao sexo masculino $(60,4 \%$ e $61,7 \%)$ e apresenta histórico de diabetes na família de $50,50 \%$ e $35,1 \%$, respectivamente.

A prevalência das doenças não transmissíveis comuns aumenta com o avançar da idade, assim como a expectativa de vida aumenta e o fardo das doenças não transmissíveis também é esperado que aumente ${ }^{(23,25)}$. Para a amostra do G1 e G2 da presente investigação, a maioria estava abaixo dos quarenta anos, pois o campus onde ocorreu o estudo participou do programa de expansão recente das universidades federais, abrindo oportunidades para novos docentes e, consequentemente, maior contratação de técnicos adminstrativos ${ }^{(21)}$.

Estudos direcionados para calcular a prevalência global inadvertidamente não calculam o predomínio dos sexos masculino e feminino, como pode ser verificado no atual estudo, pois em muitos casos essa informação não foi relatada. $\mathrm{O}$ acesso a tal informação iria reduzir o número de suposições que têm de ser feitas para os dados incompletos, e, adicionalmente, permitiria uma estimativa mais precisa ${ }^{(23)}$.

A hereditariedade é um fator de risco não modificável que eleva o risco de desenvolver DM2 em até dez vezes em relação às pessoas que não possuem histórico familiar da doença( $^{(26)}$.

A literatura fornece uma relação diretamente proporcional da prevalência de diabetes com a idade ${ }^{(27-30)}$. No Brasil, um estudo multicêntrico sobre a prevalência de DM demonstrou uma prevalência de $7,6 \%$ na população de 30 a 69 anos. Essa taxa aumentava com a idade e foi de 17,4\% no grupo etário de 60-69 anos ${ }^{(27)}$.

Um estudo com 484 policiais ativos do sexo masculino, com idade de 30 a 59 anos, foi realizado na cidade de Teresina-PI, analisando a prevalência distribuída nas faixas etárias, revelando que $3 \%$ dos diabéticos estavam na faixa etária entre 30 e 39 anos, $7 \%$ entre 40 e 49 anos e $15 \%$ entre 50 e 59 anos, reforçando que a incidência da diabetes aumenta com a idade ${ }^{(28)}$.

$\mathrm{O}$ G1 e G3 se diferenciam quando avaliado quanto à HAS $\left(17,10 \%\right.$ e $5,9 \%$ respectivamente). Em estudo prévio ${ }^{(29)}$, 
os indivíduos hipertensos ou dislipidêmicos apresentaram risco cerca de três vezes maior para o desenvolvimento do $\mathrm{DM}$, quando comparados à população sem tais fatores.

No presente estudo, docentes apresentaram o maior índice em relação à incidência de HAS. Isso pode ser devido a uma junção de fatores, como a inatividade física (59,5\%) e o estresse, o que pode culminar com a elevação da pressão arterial, pois o docente está exposto a inúmeros fatores de estresse, tornando-os uma categoria profissional de risco ${ }^{(30)}$.

Um dos fatores de destaque do presente estudo é a porcentagem de voluntários do G1 (66,66\%) e G2 (62,76\%) com sobrepeso. $\mathrm{O}$ excesso de peso, em especial a obesidade abdominal, representa um maior fator de risco para o desenvolvimento do diabetes, visto que o acúmulo do tecido adiposo está associado com a intolerância à glicose e a hiperinsulinemia. A presença da obesidade visceral abdominal parece ser o principal fator determinante da síndrome metabólica e deve ser prevenida por programas de atividade física e dieta saudável ${ }^{(31)}$.

A literatura demonstra que um dos mais comuns fatores de risco na população para DM2 é o sobrepeso, verificado principalmente mediante avaliação do índice de massa corpórea $(\mathrm{IMC})^{(31-34)}$. Em estudo prévio ${ }^{(32)}$, verificouse que $51,5 \%$ da amostra estava acima do IMC esperado. Estudo $^{(35)}$ realizado com trabalhadores industriais verificou que o excesso de peso, alteração do colesterol total e dos triglicerídeos apresentaram, respectivamente, 1,93, 1,30 e 1,88 vezes a chance de ter DM. O mesmo valor foi encontrado mediante associação da presença de obesidade, com um aumento de 1,9 na prevalência de diabetes ajustada por idade ${ }^{(27)}$.

O ideal, quanto ao status de peso, é que a pontuação obtida no presente estudo fosse zero, pois acima disso demonstra-se um risco para o desenvolvimento de DM2. Mediante análise dos dados, $66,66 \%$ do G1 apresentamse acima do peso, assim como $62,75 \%$ do G2. Fica explícita a influência do peso como fator de risco para o desenvolvimento da referida doença na população estudada.

O fator sobrepeso também se mostrou preocupante no $\mathrm{G} 3$, visto que $46,07 \%$ dos voluntários se apresentaram acima do peso. Esse valor não corresponde à maioria do grupo, mas é extremamente alto, fazendo-se necessária uma reflexão sobre o tema. Estudos ${ }^{(27,36)}$ apontam a tendência de sobrepeso em adolescentes, sugerindo que os fatores causais são alimentares e a diminuição no hábito de praticar atividade física.

No presente estudo, o sedentarismo mostrou-se um fator de risco importante para os grupos. A inatividade física atingiu a maioria dos voluntários (59,50\%) apenas no G1. Apesar de não ter atingido a maioria dos voluntários nos demais grupos (G2 e G3), os valores obtidos demonstram que uma grande parcela da população estudada não pratica atividade física regularmente. O G2 foi o mais ativo, com $38,30 \%$ dos voluntários considerados sedentários. Esse grupo possui uma característica interessante, pois os voluntários que selecionaram que eram fisicamente ativos $(38,3 \%)$ o fizeram por irem ao trabalho de bicicleta todos os dias, pedalando mais de trinta minutos nesse percurso.

A inatividade física no $\mathrm{G} 3$ foi de $41,26 \%$ dos participantes do estudo em questão. Em estudo ${ }^{(22)}$ realizado com estudantes universitários, foi verificado que o sedentarismo atingiu $33,7 \%$ dos entrevistados, índice que aumenta se forem retirados os alunos do curso de Educação Física. Isso pode ser explicado pelo fato dos mesmos apresentarem sobrecarga de horas aulas e da necessidade de dedicação para estudos e outras atividades extraclasse ${ }^{(22)}$.

Estudo realizado com funcionários públicos ${ }^{(36)}$ observou que $24,6 \%$ eram sedentários, adultos e economicamente ativos, sem diferença de sexo. Resultado menor que o encontrado no presente estudo, pois unindo os grupos de funcionários ( $\mathrm{G} 1$ e G2), tem-se $42,42 \%$ de indivíduos sedentários. Outro estudo encontrou que a maioria dos voluntários $(59 \%)$ era sedentária ${ }^{(37)}$.

Dentre os fatores pesquisados no questionário do presente estudo, apenas a obesidade e a atividade física são fatores mutáveis, ou seja, é possível diminuir o risco de desenvolver DM na população melhorando a alimentação e realizando atividade física frequentemente ${ }^{(14)}$.

A HAS também pode ser prevenida com alimentação correta e atividade física regular, mas, uma vez diagnosticada, a manutenção dos níveis pressóricos ocorre por meio de medicamentos e atividade física ${ }^{(38)}$.

Dessa forma, pode-se verificar que, para diminuir o risco de DM na população, de um modo geral, programas de atividade física devem ser idealizados e implantados, e esse tema deve fazer parte da discussão de programas de saúde pública nas universidades ${ }^{(14)}$. A prática de atividade física favorece hábitos saudáveis, causando impacto sobre o tabagismo, ingestão calórica inadequada, estresse e dependência de drogas ${ }^{(39)}$. De um modo geral, a alimentação adequada associada à prática de atividade física regular é recomendada para diminuir o risco de DM $2^{(14)}$. O exercício físico, seja o treinamento aeróbio ou de resistência muscular, desempenha um papel fundamental na prevenção e controle do DM 2 e nas complicações de saúde relacionadas a esta. Mas a prática precisa ser regular, para que os benefícios relacionados à qualidade de vida e promoção da saúde sejam atingidos $^{(39)}$.

Com o presente estudo, verificou-se que a população universitária pode ser estudada como modelo de saúde. $\mathrm{O}$ risco de desenvolver DM2 no presente estudo foi 
considerável para os docentes e funcionários da UFPI no campus de Parnaíba. Porém, o questionário utilizado deve ser extrapolado para outras populações, a fim de identificar a efetividades e a confiabilidade dele.

Os fatores mais importantes identificados como risco nessa população foram a obesidade e a inatividade física. Dessa forma, considerando as recomendações de abordagem conjunta, as informações obtidas e os limites de atuação dos serviços de saúde, ficam sugeridas as seguintes estratégias: desenvolvimento de ações de educação em saúde com ênfase na alimentação saudável, controle de peso e atividade física, sendo a monitorização do consumo alimentar e dos fatores de risco apontados como instrumentos para avaliação e subsídio para o planejamento de ações ${ }^{(40)}$.

\section{CONCLUSÃO}

Encontrou-se a prevalência do risco de desenvolver diabetes mellitus tipo 2 (DM2) de 16,21\% no grupo de docentes, $13,82 \%$ no grupo de técnicos administrativos e $1,23 \%$ no grupo de discentes. Dentre os fatores de risco mais comuns, destacaram-se a obesidade e a inatividade física.

\section{CONFLITO DE INTERESSE}

Os autores negam haver conflito de interesse referente a este artigo

\section{REFERÊNCIAS}

1. Azevedo S, Victor EG, Oliveira DC. Diabetes mellitus e aterosclerose: noções básicas da fisiopatologia para o clínico geral. Rev Bras Clin Med 2010; 8(6):520-6.

2. American Diabetes Association. Diagnosis and classification of diabetes mellitus. Diabetes Care. 2013;36(Supl 1):S67-8.

3. Rawal LB, Tapp RJ, Williams ED, Chan C, Yasin S, Oldenburg B. Prevention of Type 2 Diabetes and Its Complications in Developing Countries: A Review. Int J Behav Med. 2012;19(2):121-33.

4. International Diabetes Federation atlas update 2012 [Monografia on line]. [acesso em 2015 Abr 12]. Disponível em: http://www.idf.org/diabetesatlas/5e/ Update2012

5. Mendes TAB, Goldbaum M, Segri NJ, Barros MBA, Cesar CLG, Carandina L, et al. Diabetes mellitus: fatores associados à prevalência em idosos, medidas e práticas de controle e uso dos serviços de saúde em São Paulo, Brasil. Cad Saúde Pública. 2011;27(6):1233-43.
6. Lautt WW, Wang HH. Obesity as an early symptom of the AMIS Syndrome. J Clin Med. 2014;3(4):1178-98.

7. Dallam G, Foust CP. A Comparative Approach to using the diabetes prevention program to reduce diabetes risk in a worksite. Health Promot Pract. 2013;14(2):199204.

8. Zhang X, Luo H, Gregg EW, Mukhtar Q, Rivera $\mathrm{M}$, Baker L, et al. Obesity prevention and diabetes screening at local health departments. Am J Public Health. 2010;100(1):1434-41.

9. Lindström J, Neumann A, Sheppard KE, GilisJanuszewska A, Greaves CJ, Handke U, et al. Take action to prevent diabetes - the IMAGE toolkit for the prevention of type 2 diabetes in Europe. Horm Metab Res. 2010;42 Supl 1:37-55.

10. Buijsse B, Simmons RK, Griffin SJ, Schulze MB. Risk Assessment Tools for Identifying Individuals at Risk of Developing Type 2 Diabetes. Epidemiol Rev. 2011; 33(1):46-62.

11. Perrin B, Gardner M, Kennett S, Cornelius J, Fanning M. An organised approach to the podiatric care of people with diabetes in regional Australia. Aust Health Rev. 2012;36(1):6-21.

12. Bem AJ. Confiabilidade e análise de desempenho de dois questionários de avaliação da adesão ao tratamento anti-hipertensivo: teste de Morisky-Green e Brief Medication Questionnaire [dissertação]. Porto Alegre: Universidade federal do Rio Grande do Sul; 2011.

13. Medeiros CCM, Bessa GG, Coura AS, França ISX, Sousa FS. Prevalência dos fatores de risco para diabetes mellitus de servidores públicos. Rev Eletr Enf. 2012;14(3):559-69.

14. Sociedade Brasileira de diabetes. Diretrizes da Sociedade Brasileira de Diabetes 2013-2014. São Paulo: AC Farmacêutica; 2014.

15. Bang H, Edwards AM, Bomback AS, Ballantyne CM, Brillon D, Callahan MA, et al. Development and Validation of a Patient Self-assessment Score for Diabetes Risk. Ann Intern Med. 2009;151(11):775-83.

16. Beaton DE, Bombardier C, Guillemin F, Ferraz MB. Guidelines for the process of cross-cultural adaptation of self-report measures. Spine. 2000;25(24):3186-91.

17. Coutinho-Myrrha1 MA, Dias RC, Fernandes AA, Araújo CG, Hlatky MA, Pereira DG, et al. Duke Activity Status Index em Doenças Cardiovasculares: validação de tradução em Português. Arq Bras Cardiol. 2014;102(4):383-90. 
18. Duncan BBS, Giugliani MI, Duncan ERJ, Schmidt M, Giugliani C. Medicina Ambulatorial: condutas de atenção primária baseada em evidências. Porto Alegre: Artmed; 2013

19. Ministério da Saúde (BR), Secretaria de Vigilância em Saúde, Departamento de Análise de Situação de Saúde, Coordenação Geral de Doenças e Agravos Não Transmissíveis. Plano de ações estratégicas para o enfrentamento das doenças crônicas não transmissíveis (DCNT) no Brasil 2011-2022 [acesso em 2015 Abr 12]. Brasília: Ministério da Saúde; 2011. Disponível em: http://portal.saude.gov.br/portal/arquivos/pdf/ cartilha_plano.pdf.

20. Duncan BB, Chor D, Aquino EML, Bensenor IM, Mill JG, Schmidt MI, et al. Doenças Crônicas Não Transmissíveis no Brasil: prioridade para enfrentamento e investigação. Rev Saúde Pública. 2012;46(Supl 1):126-34

21. Associação Nacional dos Dirigentes das Instituições Federais de Ensino Superior - ANDIFES. Relatório de Acompanhamento do programa de apoio a planos de reestruturação e expansão das universidades federais (REUNI). Brasília: ANDIFES; 2010

22. Nóbrega, ECM. História familiar de doenças crônicas, atividade física e hábitos alimentares em estudantes da área da saúde. Rev Bras Promoç Saúde. 2014;27(3):33340 .

23. Cheema A, Adeloye D, Sidhu S, Sridhar D, Chan KY. Urbanization and prevalence of type 2 diabetes in Southern Asia: A systematic analysis. J Glob Health. 2014;4(1):010404

24. Reis AF, Velho G. Bases genéticas do diabetes mellitus tipo 2. Arq Bras Endocrinol Metab. 2002;46(4):426-32.

25. Malerbi DA, Franco, LJ. Multicentric study of the prevalence of diabetes mellittus and impaired tolerance in urban Brazilian population aged 30-69 yr. Diabetes Care. 1992;15(11):1509-16.

26. Martin JR, Lopez MAM, Morató TM, Kohan ID, Cuevas FJP, Jimenez RT, Puig JG. Prevalencia de diabetes en una población adulta de Madrid (España). Estudio MADRIC (MADrid RIesgo Cardiovascular). Gac Sanit. 2011;26:243-50.

27. Silva ESS. Prevalência de Diabetes Mellitus Tipo 2 em Homens de 30 a 59 anos da Polícia Militar de Teresina, Piauí [dissertação]. Rio de Janeiro (RJ): Fundação Oswaldo Cruz; 2008.

28. Souza LJ, Chalita FEB, Reis AFF, Teixeira CL, Gicovate Neto C, Bastos DA, et al. Prevalência de diabetes mellitus e fatores de risco em Campos de Goyatacazes, RJ. Arq Bras Endocrinol Metab. 2003;47(1):69-74.

29. Reinhold HH. O sentido da vida: prevenção de stress e burnout do professor [tese]. Campinas: PUC; 2004.

30. Collins GS, Mallett S, Omar O, Yu LM. Developing risk prediction models for type 2 diabetes: a systematic review of methodology and reporting. BMC Med. 2011;9:103-17.

31. Ortiz MCA, Zanetti ML. Levantamento dos fatores de risco para diabetes mellitus tipo 2 em uma instituição de ensino superior. Rev Latinoam Enferm. 2001;9(3):5863.

32. Mazzini MCR, Blumer MG, Hoehne EL, Guimarães KRLSLQ, Caramelli B, Fornari L, et al. Rastreamento do risco de desenvolvimento de diabetes mellitus em pais de estudantes de uma escola privada na cidade de Jundiaí, São Paulo. AMB Rev Assoc Med Bras. 2013;59(2):136-42.

33. Martinz MC, Latorre MRDO. Fatores de risco para hipertensão arterial e diabete melito em trabalhadores de empresa metalúrgica e siderúrgica. Arq Bras Cardiol. 2006;87(4):471-9.

34. Pinho L, Flávio EF, Santos SHS, Botelho ACC, Caldeira AP. Excesso de peso e consumo alimentar em adolescentes de escolas públicas no norte de Minas Gerais, Brasil. Ciênc Saúde Coletiva. 2014;19(1):6774.

35. Cortês DCS, Paula R, Mendonça APP, Torres PRR, Arantes AA, Leal AB, et al. Sedentarismo em população específica de funcionários de uma empresa pública. Rev Bras Clin Med. 2012;8(5):375-7.

36. Molena-Fernandes CA, Ferrari A, Netto-Oliveira ER, Oliveira AAB. Associação entre sobrepeso e obesidade e fatores de risco cardiovascular em funcionários de uma empresa de seguro-saúde. Rev Educ Fís. 2006;17(1):75-83.

37. Simão AF, Precoma DB, Andrade JP, Correa Filho H, Saraiva JFK, Oliveira GMM, et al. I Diretriz Brasileira de prevenção cardiovascular. Arq Bras Cardiol. 2013;101(Supl 2):1-63.

38. VI Diretrizes Brasileiras de Hipertensão. Arq Bras Cardiol. 2010;95(1 Supl 1):1-51.

39. Colderg SR, Sigal RJ, Fernhall B, Regensteiner JG, Blissmer BJ, Rubin RR, et al. Exercise and Type 2 Diabetes. The American College of Sports Medicine and the American Diabetes Association: joint position statement. Diabetes Care. 2010;33(12):147-67. 
40. Raddatz, A, Scholze AS, Duarte Júnior CF, Silveira PFA. Análise do discurso da Política Nacional de Promoção da Saúde. Rev Bras Promoç Saúde. 2011;24(3):191-8.

\section{Endereço do primeiro autor:}

Alessandra Tanuri Magalhães

Universidade Federal do Piauí, campus Ministro Reis Veloso

Avenida São Sebastião, 2819

Bairro: Nossa Senhora de Fátima

CEP: 64202-020 - Parnaíba - PI - Brasil

E-mail: alessandra@ufpi.edu.br
Endereço para correspondência:

Vinicius Saura Cardoso.

Universidade Federal do Piauí, campus Ministro Reis Veloso

Avenida São Sebastião, 2819

Bairro: Nossa Senhora de Fátima

CEP: 64202-020 - Parnaíba - PI - Brasil

E-mail: vscfisio@ufpi.edu.br 